\title{
Quand le NGS aide ò résoudre une énigme diagnostique
}

\author{
Emmanuelle Lagrue, Brigitte Gilbert-Dussardier, Frédéric Bilan
}

\begin{abstract}
L'amyotrophie spinale proximale prédominant aux membres inférieurs (ou SMA-LED pour spinal muscular atrophy with lower extremities predominance) est une forme rare de SMA, le plus souvent secondaire à une mutation du gène DYNC1H1. Ce gène code pour les chaines lourdes du complexe dynéine responsable du transport rétrograde de la synapse vers le corps cellulaire neuronal. Le spectre phénotypique des mutations du gène DYNC1H1 est vaste, mais l'association d'une pathologie neurologique périphérique (SMA-LED ou maladie de Charcot Marie Tooth de type 2) à une atteinte neurologique centrale (déficience cognitive avec ou sans malformation corticale) peut guider le clinicien.
\end{abstract}

\section{Observation}

Le jeune M., âgé de 5 ans, est le troisième enfant d'une fratrie de trois, issu de parents jeunes, non apparentés (mère d'origine française, père d'origine franco-américaine), et sans antécédents familiaux. Il n'est pas noté de difficultés conceptionnelles. L'accouchement a été eutocique, au terme d'une grossesse physiologique. La biométrie de naissance était normale. Un décalage de développement a été noté à partir du deuxième semestre de vie avec : des retournements dorso-ventral et ventro-dorsal acquis à 9-10 mois, une station assise stable à 12 mois, une mise assis à 15 mois, une marche libérée à 31 mois, et une absence de langage à 36 mois. Ce tableau de déficience intellectuelle globale amène à une évaluation en génétique à l'âge de 3 ans. L'examen clinique est alors peu contributif. On note néanmoins des particularités faciales (fentes palpébrales orientées en bas et dehors, nez bulbeux, narines antéversées, philtrum long, lèvre supérieure fine, oreilles de grande taille) mais qui n'orientent pas vers une origine génétique donnée. Des investigations paracliniques sont alors décidées et comprennent un bilan morphologique (fond d'œil et lampe à fente, échographie abdominale, IRM cérébrale), un dosage des enzymes musculaires, une recherche de fragilité de l'X, et une CGH-array pour dépister d'éventuels remaniements chromosomiques. Ces examens mettront en évidence une myopie à -7 dioptries et une bifidité rénale, sans pouvoir établir l'étiologie de la déficience cognitive. L'évolution se fait secondairement vers l'apparition d'une fatigabilité musculaire avec des chutes notées à partir de 3 ans et demi, ces nouveaux éléments motivant une demande d'évaluation neuromusculaire dans notre centre de référence. Lors de la première consultation, l'examen morphologique retrouve les particularités faciales déjà connues. L'auscultation cardiopulmonaire, la palpation abdominale, l'aspect des organes génitaux et de la peau sont habituels. Sur le plan neuromoteur, il existe une amyotrophie marquée des quadriceps et ischio-jambiers ainsi qu'une amyotrophie plus modérée des mollets. On ne note pas de myotonie, d'atteinte faciale, ou de phénomène de rippling (Figure 1). On note un syndrome pyramidal des membres inférieurs (hypertonie spastique, réflexes rotuliens et achilléens vifs, polycinétiques, et trépidation épileptoïde). Dans le contexte de suspicion de maladie neuromusculaire, les investigations sont reprises. Les IRM médullaire et musculaire, l'échographie cardiaque et les épreuves fonctionnelles respiratoires s'avèrent normales. De même, l'EMG ne montre pas d'anomalie ni en neurographie, ni en myographie, et il n'y a pas non plus d'atteinte de la jonction neuromusculaire. Du fait de l'impasse diagnostique, et dans l'hypothèse d'une maladie d'origine génétique, une étude en NGS (séquençage à haut débit de l'exome entier) est alors demandée chez le propositus et ses deux parents (étude de trio). La présence à l'état hétérozygote de la variation $\mathrm{c} 3188 \mathrm{C}>\mathrm{T}$ au niveau de l'exon 13 du gène DYNC1H1 permet d'affirmer le diagnostic de SMALED. La mutation est survenue de novo, n'est pas répertoriée dans les bases de données, et est considérée comme pathogène par les logiciels de prédiction.

\section{Commentaire}

Le rôle principal du complexe dynéine est le transport rétrograde (de la synapse vers le corps cellulaire neuronal) des organelles, y compris les mitochondries [1]. Ses rôles complémentaires sont multiples : organisation du fuseau mitotique, positionnement du réticulum endoplasmique, de l'appareil de Golgi 


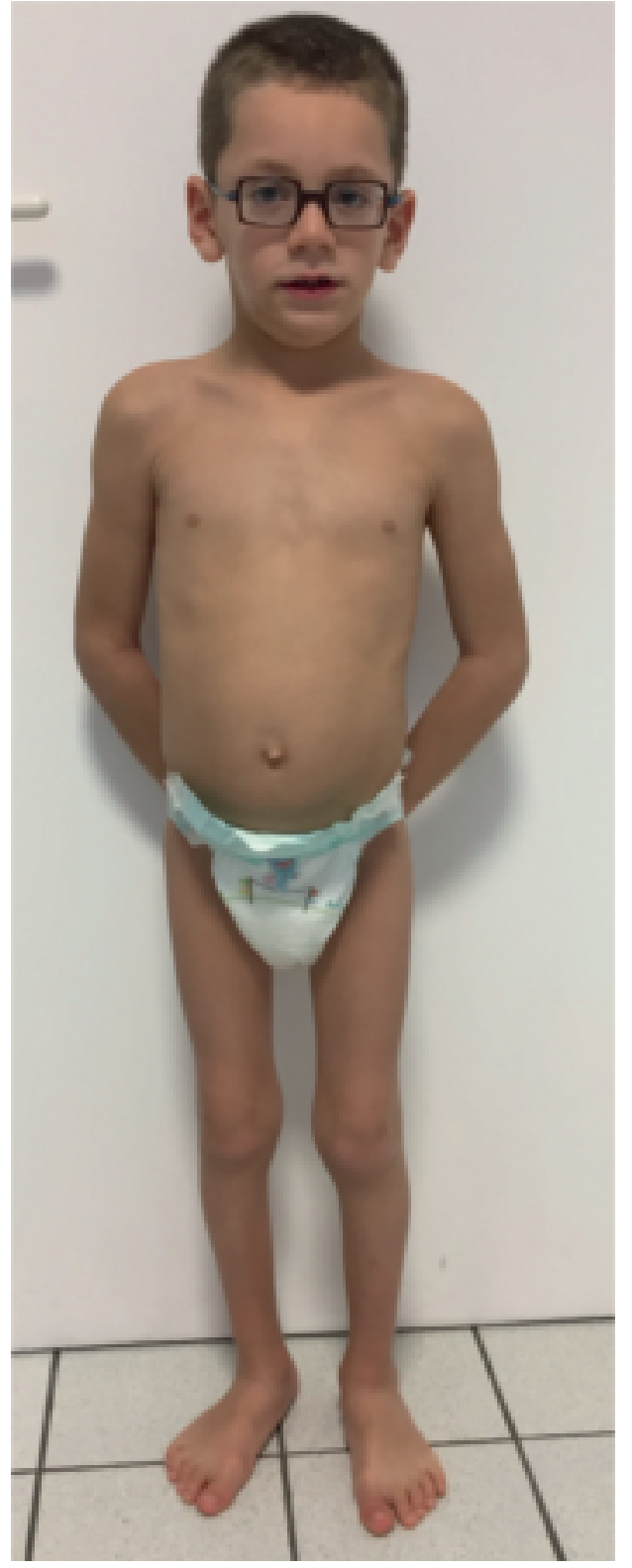

Figure 1

Aspect de SMA-LED avec amyotrophie marquée des membres inférieurs atteignant l'ensemble de l'axe jambier (quadriceps, ischio-jambiers, gastrocnémiens).

Des particularités faciales sont également notées chez notre patient.

et du noyau dans le corps cellulaire, autophagie et maintenance des endosomes. Le gène DYNC1H1 code les chaînes lourdes du complexe dynéine, qui s'homodimérisent en $\mathrm{N}$ terminal (tail domain) et recrutent les chaines intermédiaires, légères intermédiaires et légères, responsables quant à elles de la sélectivité pour les molécules transportées (Figure 2) [2]. DYNC1H1 constitue donc un gène primordial au sein du système nerveux central et périphérique, expliquant la variabilité phénotypique en cas de mutation. DYNC1H1 est ainsi impliqué chez l'homme dans la survenue de malformations corticales, dans une forme axonale de CMT (CMT 2O) et dans des cas de SMA-LED. Il est intéressant de noter que d'autres gènes intervenant dans le transport rétrograde, tel que ceux codant des kinésines, ont déjà été impliqués dans des phénotypes similaires [3, 4].

Concernant les modèles animaux, on observe que le knock-out homozygote de DYNC1H1 n'est pas viable chez la souris en raison d'un trouble massif du développement cérébral. En cas de mutation stop localisée hors du domaine de dimérisation des chaines lourdes (laquelle entraine traditionnellement un CMT axonal chez l'homme), les atteintes sont variées avec trois modèles distincts. La souris Loa (legs at odd angles) présente une atteinte des neurones moteurs et sensitifs proprioceptifs [5], tandis que la souris Cra1 (Cramping1) a des jonctions neuromusculaires pathologiques [6], et que la souris $S w l$ (Sprawling) présente une neuropathie sensitive et une perte des fuseaux neuromusculaires [7], ce qui en fait des modèles robustes en recherche préclinique. La situation est plus complexe pour le modèle murin SMA-LED. Les souris avec mutation ponctuelle dans le tail domain de DYNC1H1 présentent en effet l'association d'une atteinte périphérique (de type sensitive) et centrale, mais cette atteinte centrale est non corticale (atteinte striatale). Ce modèle murin de SMA-LED a néanmoins pu apporter des informations nouvelles, en particulier sur la présence d'une atteinte mitochondriale. Il a ainsi été prouvé la survenue d'altérations morphologiques mitochondriales et d'une perte de la mitofusine 1 en particulier, soulignant là encore la physiopathologie commune avec les CMT axonaux par mutation du gène MFN2 [8].

Chez l'homme, la publication princeps de Weedon etal. [9] a prouvé en 2011 l'implication de DYNC1H1 dans une large famille multiplex comprenant 23 patients sur quatre générations atteints de CMT axonal. Le phénotype rapporté était celui d'un CMT "classique" (décalage des acquisitions motrices initiales, atteinte distale avec pieds creux, atteinte rare aux membres supérieurs, maintien d'une ambulation à l'âge adulte) avec néanmoins des éléments inhabituels chez certains sujets, tel que la persistance des réflexes ou de la proprioception, une prédominance proximale voire scapulaire du déficit moteur, ou enfin des éléments cérébelleux. Un an plus tard, Harms et al. [10] élargit le phénotype aux SMA-LED à transmission dominante, avec comme éléments phénotypiques un début dans l'enfance, voire congénital, une atteinte 


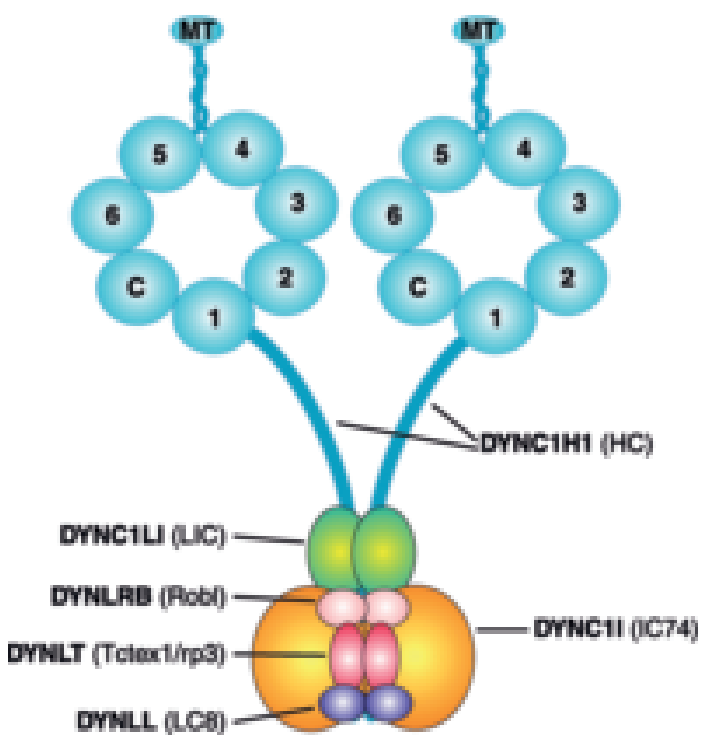

Figure 2

Structure du complexe dynéine selon Pfister et al. (PLos Genet, 2006).

L'extrémité C-terminale représente le domaine moteur d'hydrolyse d'ATP. Les chaines lourdes codées par DYNC1H1

s'homodimérisent à l'extrémité $\mathrm{N}$-terminale où elle se lient aux autres chaines du complexe (codées par DYNC1LI, DYNC1I, $D Y N L R B, D Y N L T$, et $D Y N L L)$

à prédominance proximale, peu évolutive dans le temps, et associée dans $30 \%$ des cas à une déficience intellectuelle sans malformation corticale. L'association à de possibles malformations corticales (le plus souvent de type polymicrogyrique) sera ensuite validée dans d'autres observations [11, 12], tout comme l'implication du premier motoneurone avec présence d'une paraparésie spastique, comme observé chez notre patient [13]. Enfin, on notera la coexistence possible à une cataracte plus récemment décrite, qu'elle soit congénitale [14] ou acquise [15].

En conclusion, le phénotype des dynéinopathies par mutation du gène DYNC1H1 ne cesse de s'élargir. Dans le contexte du SMA-LED, il faut savoir suspecter une telle étiologie devant l'association d'une pathologie neuromusculaire neurogène, prédominant volontiers aux membres inférieurs, le tout associé à une atteinte centrale. Rappelons que d'autres gènes de SMA-LED ont été rapportés comme le gène BICD2 et que de façon générale, de tels tableaux cliniques peuvent rentrer dans le champ plus vaste des amyotrophies spinales distales. Si besoin en était, cette observation prouve à nouveau l'intérêt et la puissance du NGS dans la résolution de situations diagnostiques difficiles.

Solving a diagnostic riddle with NGS

\section{LIENS D'INTÉRÊT}

Les auteurs déclarent n'avoir aucun lien d'intérêt concernant les données publiées dans cet article.

\section{RÉFÉRENCES}

1. Hafezparast M, Klocke R, Ruhrberg C, et al. Mutations in dynein link motor neuron degeneration to defects in retrograde transport. Science 2003 ; $300: 808-12$.

2. Pfister KK, Shah PR, Hummerich H, et al. Genetic analysis of the cytoplasmic dynein subunit families. PLoS Genet 2006 ; 2 : e1.

3. Gentil BJ, Cooper L. Molecular basis of axonal dysfunction and traffic impairments in CMT. Brain Res Bull 2012; 88 : 444-53. 4. Poirier K, Lebrun N, Broix L, et al. Mutations in TUBG1, DYNC1H1, KIF5C and KIF2A cause malformations of cortical development and microcephaly. Nat Genet 2013 ; 45 : 639-47. 5. Deng W, Garrett C, Dombert, et al. Neurodegenerative mutation in cytoplasmic dynein alters its organization and dyneindynactin and dynein-kinesin interactions. J Biol Chem 2010 285 : 39922-34

6. Courchesne SL, Pazyra-Murphy MF, et al. Neuromuscular junction defects in mice with mutation of dynein heavy chain 1 . PLoS One 2011 ; 6 : e16753.

7. Chen XJ, Levedakou EN, Millen KJ, et al. Proprioceptive sensory neuropathy in mice with a mutation in the cytoplasmic Dynein heavy chain 1 gene. J Neurosci 2007 ; 27 : 14515-24.

8. Burté F, Carelli V, Chinnery PF, et al. Disturbed mitochondrial dynamics and neurodegenerative disorders. Nat Rev Neurol $2015 ; 11: 11-24$

9. Weedon MN, Hastings R, Caswell R, et al. Exome sequencing identifies a DYNC1H1 mutation in a large pedigree with dominant axonal Charcot-Marie-Tooth disease. Am J Hum Genet 2011; 89 : 308-12

10. Harms MB, Ori-McKenney KM, Scoto M, et al. Mutations in the tail domain of DYNC1H1 cause dominant spinal muscular atrophy. Neurology 2012; 78 : 1714-20.

11. Fiorillo C, Moro F, Yi J, et al. Novel dynein DYNC1H1 neck and motor domain mutations link distal spinal muscular atrophy and abnormal cortical development. Hum Mutat 2014; 35 : 298-302.

12. Scoto M, Rossor AM, Harms MB, et al. Novel mutations expand the clinical spectrum of DYNC1H1-associated spinal muscular atrophy. Neurology $2015 ; 84$ : 668-79.

13. Strickland AV, Schabhüttl M, Offenbacher $\mathrm{H}$, et al. Mutation screen reveals novel variants and expands the phenotypes associated with DYNC1H1. J Neurol 2015; 262 : 2124-34.

14. Gelineau-Morel R, Lukacs M, Weaver KN, et al. Congenital cataracts and gut dysmotility in a DYNC1H1 dyneinopathy patient. Genes (Basel) 2016; $7: 85$.

15. Hertecant J, Komara M, Nagi A, et al. A novel de novo mutation in DYNC1H1 gene underlying malformation of cortical development and cataract. Meta Gene 2016 ; 9 : 124-7. 